INNATE IMMUNITY

\section{APOL1 variants contribute to racial disparity in sepsis}

Individuals of African ancestry are more likely to die from sepsis than individuals of European ancestry and

mitochondrial defects in G2APOL 1 cells are linked to the inflammatory endothelial phenotype as a result of leakage of mtDNA into the cytosol

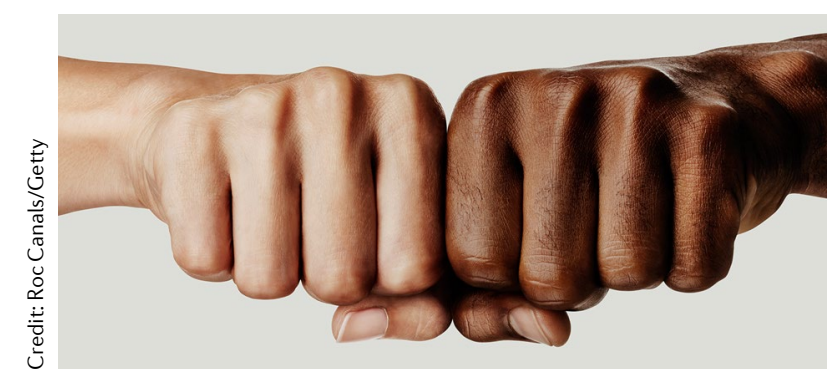

of endothelial dysfunction and vascular damage. The authors suggest that both the APOL1 risk genotype and the increase in APOL1 expression are critical for disease development.

To explore the role of APOL1 in endothelial dysfunction, the authors generated mice in which expression of a human APOL1 risk allele (G2APOL1) or wild-type allele is induced specifically in endothelial cells. Endothelial expression of G2APOL1 was associated with loss of glomerular capillary fenestrations and delamination of alveolar capillaries, and led to increased vascular permeability. Moreover, transcripts for endothelial glycocalyx remodelling (an indicator of endotheliopathy), vascular inflammation and adhesion were upregulated in mice expressing G2APOL1.

Consistent with a role in sepsis, mice with endothelial G2APOL1 showed higher mortality following lipopolysaccharide (LPS)-induced endotoxaemia or caecal ligation and puncture, suffering more severe end-organ damage, kidney disease and lung damage compared with control mice. The more severe disease in G2APOL1-expressing mice was associated with higher expression of pro-inflammatory proteins and markers of endothelial damage. Indeed, single-cell sequencing revealed 534 differentially expressed genes in endothelial cells of G2APOL1 versus wild-type mice; notably, interferon-signature genes were upregulated and cell junction genes were downregulated in cells expressing the G2APOL1 allele.

So, what links endothelial G2APOL1 to the inflammatory phenotype? Microscopic examination showed potential defects in mitochondria and mitophagy in G2APOL1 endothelial cells.
Indeed, a mitophagy reporter gene indicated lower basal mitophagy and starvation-stimulated mitophagy, leading to an accumulation of mitochondria in G2APOL1 endothelial cells. Other defects associated with G2APOL1 expression included reduced mitochondrial function, loss of mitochondrial negative membrane potential and attenuated autophagy.

So, the authors hypothesized that the mitochondrial defects in G2APOL1 cells are linked to the inflammatory endothelial phenotype as a result of leakage of mtDNA into the cytosol. Consistent with this, analysis of the cytosolic fraction indicated higher mtDNA content in G2APOL1 endothelial cells than wild-type cells. Moreover, G2APOL1 cells showed activation of the cytosolic DNA sensing NLRP3 inflammasome and cGAS-STING pathways. Depletion of mtDNA using ethidium bromide treatment reduced this activation, and knockdown of Cgas and Sting mRNAs prevented induction of the downstream inflammatory genes.

Finally, the authors tested the role of this pathway in sepsis. Genetic deletion of Nlrp3, Gsdmd, Casp1 and Sting rescued the endothelial barrier defect of G2APOL1 endothelial cells in vitro and in vivo. And treatment of G2APOL1 ${ }^{+}$mice with small-molecule inhibitors of caspase 1, gasdermin D and STING reduced the weight loss, inflammatory response, end-organ damage and mortality following LPS-induced sepsis.

These data show that the APOL1 risk genotype is a key determinant of sepsis - through mitochondrial dysfunction and pro-inflammatory endothelial changes - and could explain an important racial disparity observed in sepsis incidence and severity amongst individuals of African ancestry.

Lucy Bird

ORIGINAL ARTICLE Wu, J. et al. APOL1 risk variants in individuals of African genetic ancestry drive endothelial cell defects that exacerbate sepsis. Immunity https://doi.org/10.1016/j.immuni. 2021.10.004 (2021) 